\section{A tiny colony-forming Staphylococcus aureus from a clinical specimen which grew better on MacConkey's medium}

An ear swab from a patient with otitis externa was cultured on blood agar and MacConkey's plates. Both were incubated aerobically at $37^{\circ} \mathrm{C}$. Another blood agar plate, inoculated with the specimen, was also incubated anaerobically. After overnight incubation there was scanty growth of tiny $(0 \cdot 2-0 \cdot 3 \mathrm{~mm})$ translucent colonies on both blood agar plates; but the MacConkey's plate produced mediumsized (about $1 \mathrm{~mm}$ ), opaque, pinkishwhite colonies. Gram staining from all three plates showed Gram-positive cocci in clusters. Standard laboratory tests, ${ }^{1}$ including coagulase, DNAse, and mannitol fermentation, established that the growths from all three plates were Staphylococcus aureus. By disc-diffusion test the organism was found to be sensitive to all commonly used antibiotics, including penicillin and gentamicin. On repeated subcultures the organism continued to produce tiny translucent colonies on nutrient agar, blood agar, and chocolate agar plates even after 48 hours' incubation in the presence or absence of carbon dioxide, aerobically or anaerobically. On all occasions MacConkey's medium produced better growth with mediumsized opaque colonies.

To exclude the possibility of the presence of any inhibitory substances in our ordinary media, a control strain of Staph aureus (Oxford strain) was cultured in parallel with the test strain on blood agar and MacConkey plates. The control strain produced large (about $3 \mathrm{~mm}$ ) opaque colonies on the blood agar and medium-sized (about $1 \mathrm{~mm}$ ) colonies on MacConkey, while the test strain produced two different types of colonies on two plates, as before. This colonial character of the test strain and the fact that it was Staph aureus were also confirmed by a neighbouring laboratory. To see whether any of the constituents of MacConkey's medium (not present in blood agar) were responsible for the improved growth of this strain, cultures were made on specially prepared blood agar plates containing any one of neutral red, lactose, or bile salt, in the same concentrations as are used in MacConkey's medium. The plates were examined after incubation at $37^{\circ} \mathrm{C}$ for 24 hours and 48 hours, and all produced exactly similar tiny colonies; only MacConkey's medium produced better growth. It was thus proved that the MacConkey's medium was better for supporting this particular strain than other commonly used media, and that none of the constituents of MacConkey's medium could improve the growth when used alone in the ordinary media.

Small or 'dwarf' colony-forming Staph aureus have been described in the literature. ${ }^{2-4}$ They are usually produced under inhibitory conditions and tend to revert to their usual size and growth character when such conditions are removed. 5 The strains studied by Lacey and Mitchell $^{34}$ were highly pigmented ones and resistant to aminoglycosides. The strain described here was different from those of other authors in many ways: (a) it grew better on an inhibitory medium such as MacConkey's than on the usual non-inhibitory media; (b) tiny colonies produced on blood agar were translucent and not pigmented; (c) it was sensitive to antibiotics; $(d)$ repeated subcultures failed to improve the size of the colonies.

The clinical implication of this finding is that such organisms are likely to be missed or ignored if this finding is not borne in mind. Had it not grown better on MacConkey's, the scanty growth of tiny colonies on the blood agar plate from an ear swab might have been ignored in this case.

M RAHMAN Department of Microbiology, King's Mill Hospital, Sutton-in-Ashfield, Notts NG17 4JL

\section{References}

${ }^{1}$ Cowan ST. Cowan and Steel's manual for the identification of medical bacteria. London: Cambridge University Press, 1974;47-50.

${ }^{2}$ Hale JH. Studies on staphylococcal mutation: a naturally occurring " $G$ " gonidial variant and its carbon-dioxide requirement. Br J Exp Pathol 1951;32: 307-13.

${ }^{3}$ Lacey RW. Dwarf-culony variants of Staphylococcus aureus resistant to aminoglycoside antibiotics and to a fatty acid. $J$ Med Microbiol 1969;2:187-96.
Lacey RW, Mitchell AAB Gentamicinresistant Staphylococcus aureus. Lanc't $1969 ;$ ii : $1425-6$

Wilson GS, Miles A. Topley and Wilson's principles of bacteriology, virology and immunity. London: Edward Arnold Ltd, @ 1975;764-801

\section{Blood thixotropy}

In the Journal of Clinical Pathology, May 1980 (p 418), J Stuart and M Kenny reported that "blood, like non drip thixotropic paint, shows a fall in viscosity with time during shearing'. This has not been our experience. At a constant shear stress of $1.73 \mathrm{Nm}^{2}$ we observed a variation of shear rates within $1 \%$ over a period of 2 hours. The only occasions when a thixotropic-like behaviour has been demonstrated were when blood was allowed to cool before shearing at $37^{\circ} \mathrm{C}$, or allowed to stand before shearing. In the first case, the decrease in viscosity can be attributed to a simple temperature effect; in the second, from a breakup of preformed rouleaux. It may be argued that the thixotropic nature of blood is therefore an artefact of insufficient mixing.

If the breakup of a rouleaux formation is to be defined as thixotropy, then, at low shearing, blood also shows rheopexy as rouleaux formation begins. Using a couette system linked to a Deer Rheometer, we observed that at a shear stress of 0.86 $\mathrm{Nm}^{-2}$ or less, sedimentation occurred, resulting in a steady increase in viscosity over a period of time. If at the end of this period blood was sheared at a higher rate the viscosity returned to its original level. When an insufficiently mixed sample is first sheared at a low stress the resulting viscosity is extraneously high, and, because the degree of separation is virtually impossible to quantify, reproducibility must also be poor.

We believe that it is perfectly acceptable to use one sample for a whole shear range. but, first, shearing must be carried out at a high rate before low shear rate readings are taken, and not the reverse as reported. In practice, we find that after shearing at $1.73 \mathrm{Nm}^{-2}$ we can take reproducible readings at progressively lower shear over a time limit of 10 minutes before it is necessary to 'remix' at the higher shear stress. 
It has also been reported that with the use of capillary viscometers blood is slightly thixotropic, but we doubt whether this could be demonstrated in a rotational viscometer provided the blood was well mixed beforehand.

P DAVENPORT S ROATH

South Laboratory and Pathology Block, Southampton General Hospital, Tremona Road, Southampton SO9 $4 X Y$

Professor Stuart and Dr Kenny reply as follows:

It has been reported on several occasions that viscosity measurements on human blood are dependent on both time and rate of shear. ${ }^{1-5}$ Using a Contraves Low-Shear 30 viscometer, we have exposed normal blood to increasing shear rate over the range $0-1.0 \mathrm{mPas}$. The resulting viscosity tracing (or rheogram) showed a progressive decrease in viscosity as shear rate increased. When the process was reversed, so that the same specimen was exposed to progressively decreasing shear, the reverse tracing did not follow the original curve but showed a lower viscosity at all shear rates, indicating an alteration in the characteristics of the blood. This phenomenon, probably a consequence of red cell disaggregation, is consistent and reproducible and demonstrates that viscosity is time and shear-rate dependent under these experimental conditions of low shear.

The variable shear-stress instrument used by Davenport and Roath works on a different principle and will require to be evaluated in its own right. We estimate, however, that the shear-rate range produced by the shear stresses stated is much higher than our own so that rouleaux would be dispersed. Few rheologists would support the suggestion that a viscometer should first be operated at high shear in order to 'mix' blood before readings at low shear are made. As the authors seem to have demonstrated, this technique will disperse rouleaux for several minutes. Yet the effect of plasma proteins in causing rouleaux formation at low shear is probably one of the most important determinants of hyperviscosity in patients with vascular disease.

Rheologists are, of course, aware that temperature is a critical determinant of viscosity and must be rigorously controlled. Adequate mixing, but not shearing, of blood before testing is also essential, and even when a well-mixed specimen has been placed in the viscometer cup, the instrument should begin rotation within 60 seconds to avoid false-low readings at low shear rates as a consequence of red cell settling (Inglis, Carson, and Stuart, unpublished).

\section{J STUART \\ MW KENNY \\ Department of Haematology, Medical School, University of Birmingham, Birmingham B15 2TJ}

\section{References}

${ }^{1}$ Dintenfass L. Thixotropy of blood at very low rates of shear. Kolloidzeitschrift 1962;180:160.

2 Dintenfass L. Thixotropy of blood and proneness to thrombus formation. Circ Res 1962;11 :233-9.

${ }^{3}$ Dintenfass L. Study in thixotropy of concentrated pigment suspensions. Part II. Thixotropic recovery and nonequilibria states. Rheol Acta 1962;2: 187-202.

${ }^{4}$ Huang CR, Fabisiak W. Thixotropic parameters of whole human blood. In: Copley AL, Okamoto S, eds. Hemorheology and Thrombosis. Proceedings of the United States-Japan Cooperative Seminar, Kobe, Japan. New York: Pergamon Press, 1976;1-8.

5 Dintenfass L. Rheology of Blood in Diagnostic and Preventive Medicine. London: Butterworths, 1976.

\section{Book reviews}

Clinics in Haematology. Vol 9. No. 2. 'The Porphyrias.' Ed A Goldberg and MR Moore. (Pp 226; illustrated; £9.) WB Saunders. 1980.

The porphyrias are a fascinating group of disorders, which impose themselves on all clinical specialties. This monograph is particularly timely because during the past few years the enzyme defect has been demonstrated in at least three of these diseases, our understanding of the pathogenesis of the clinical syndromes has been significantly increased, and there have been useful methodological developments.

In the first chapter Dr Moore takes us gently but thoroughly through the biosynthesis of porphyrins. The acute and cutaneous porphyrins are fully discussed in the subsequent two chapters. Variegate porphyria is reviewed by Dr Kramer, and recent studies on the neurotoxicity of amino-laevulinic acid are described. The unique experience of Professor Ippen's group with congenital porphyria include new clinical observations. An interesting chapter by $\mathrm{Dr}$ With on porphyrias in animals and an important contribution on the clinical chemistry of the porphyrias by Professor Elder are followed by a section on drugs and hepatic porphyrias and a discussion of abnormalities of porphyrin metabolism in diseases other than the porphyrias.

This is an excellent monograph; the contributors reflect Who's Who of the porphyrias, and even George III would be pleased! Perhaps the use of consistent nomenclature throughout the various chapters would have been helpful but nevertheless it is a highly recommended book.

TJ PETERS

Medical Information Systems. Ralph R Grams. (Pp 464; 114 Figs + 40 charts; \$49.50.) The Humana Press Inc. 1979.

Readers of this book will have much to interest them concerning the definition and analysis of the problems that face a clinical pathology department introducing a computer into its management. Nevertheless since the system described in this 